\title{
Prediction and perinatal management of severely restrictive atrial septum in fetuses with critical left heart obstruction: Clinical experience using pulmonary venous Doppler analysis
}

\author{
Allison Divanović, MD, Kan Hor, MD, James Cnota, MD, Russel Hirsch, MD, \\ Meredith Kinsel-Ziter, RDCS, and Erik Michelfelder, MD
}

\begin{abstract}
Objective: Up to $20 \%$ of fetuses with critical left heart obstructive lesions have highly restrictive or intact atrial septae. Although this condition is generally tolerated in utero, severe hypoxemia requiring emergency atrial septostomy often develops in newborns with restrictive atrial septum. We have reported that a pulmonary venous Doppler forward/reverse time-velocity integral ratio less than 5 is highly predictive of the need for emergency atrial septostomy. We reviewed our subsequent experience using fetal pulmonary venous Doppler patterns to identify and manage fetuses with critical left heart obstruction and suspected restrictive atrial septum.
\end{abstract}

\begin{abstract}
Methods: A retrospective review of neonates with a prenatal diagnosis of critical left heart obstruction was performed. Fetal restrictive atrial septum was defined as a small/absent interatrial shunt on 2-dimensional imaging and a mean forward/reverse time-velocity integral ratio of 5 or less. Available serial pulmonary venous Doppler data were reviewed. The primary outcome was postnatal confirmation of restrictive atrial septum or severe left atrial hypertension.
\end{abstract}

Results: Eight of 39 infants had a forward/reverse time-velocity integral ratio of 5 or less. A restrictive atrial septum was confirmed postnatally in 6 of 8 infants. Overall, a forward/reverse time-velocity integral ratio of 5 or less had a sensitivity of $100 \%$ and specificity of $94 \%$ for emergency atrial septostomy. Lowering the cutoff value to 3 or less would have eliminated false-positive diagnoses in the current series. Serial data demonstrated that late second trimester values did not change in later gestation with respect to either threshold in 30 of 32 fetuses.

Conclusions: In the fetus with critical left heart obstruction, a threshold forward/reverse time-velocity integral ratio of 3 or less optimizes specificity for predicting emergency atrial septostomy. Most late second trimester values will not change over time with regard to threshold levels. (J Thorac Cardiovasc Surg 2011;141:988-94)

The incidence of hypoplastic left heart syndrome is estimated at 0.16 to 0.36 per 1000 live births, with $6 \%$ to $20 \%$ of those infants found to have a restrictive or intact atrial septum (RAS). ${ }^{1-4}$ Although this is generally well tolerated in utero, these infants often experience severe postnatal hypoxemia and left atrial hypertension, which can quickly lead to cardiovascular collapse if an emergency atrial septostomy (EAS) is not performed. Prenatal identification of such infants is important because it often affects counseling and the delivery strategy of these mothers. In the current era of fetal therapy, some centers have performed cesarean sections $(\mathrm{C} / \mathrm{S})$ in a pediatric operating room or hybrid catheterization laboratory to further shorten the interval

\footnotetext{
From the Fetal Heart Program, Division of Cardiology, Cincinnati Children's Hospital Medical Center, Cincinnati, Ohio.

Disclosures: Authors have nothing to disclose with regard to commercial support.

Received for publication March 9, 2010; revisions received Aug 27, 2010; accepted for publication Sept 9, 2010; available ahead of print Dec 6, 2010.

Address for reprints: Allison Divanović, MD, Assistant Professor, Department of Pediatrics, Division of Cardiology, Cincinnati Children's Hospital Medical Center, 3333 Burnet Avenue, MLC 2003, Cincinnati, OH 45229 (E-mail: Allison. Divanovic@cchmc.org).

0022-5223/\$36.00

Copyright (C) 2011 by The American Association for Thoracic Surgery doi:10.1016/j.jtcvs.2010.09.043
}

between birth and EAS. ${ }^{5,6}$ Select centers have even performed in utero therapies to decompress the left atrium and allow for left to right shunting. ${ }^{1-3,7-9}$ In this setting, accurate prenatal diagnosis of RAS in infants with critical left heart obstruction (CLHO) is critical in patient selection for fetal cardiac interventions or a maternal $\mathrm{C} / \mathrm{S}$ to ensure timely intervention on the atrial septum.

Prenatal diagnosis of a restrictive atrial septum takes into account multiple factors, including the 2-dimensional appearance of the septum, gradient across the interatrial shunt, and pulmonary venous Doppler (PVD) flow patterns. Pulsed PVD flow patterns have been assessed both in normal fetuses and in fetuses with CLHO. ${ }^{10-16}$ We reported in 2005 that a PVD forward/reverse velocity time integral (FR VTI) ratio less than 5 was highly predictive-maximizing both sensitivity and specificity-of the need for EAS in the fetus with CLHO. ${ }^{12}$

The purpose of this study was to (1) review our subsequent experience using fetal PVD patterns (specifically, an FR VTI threshold value $<5$ ) in identification and perinatal management of fetuses with CLHO and RAS; and (2) evaluate longitudinal variation of PVD flow indices between late second and third trimester fetal assessments. 


\section{Abbreviations and Acronyms \\ $\mathrm{CLHO}=$ critical left heart obstruction \\ $\mathrm{C} / \mathrm{S}=$ cesarean section \\ EAS = emergency atrial septostomy \\ ECMO = extracorporeal membrane oxygenation \\ EXIT = ex utero intrapartum therapy \\ FR VTI $=$ forward/reverse velocity time integral \\ PVD = pulmonary venous Doppler \\ RAS = restrictive or intact atrial septum}

\section{MATERIALS AND METHODS \\ Study Population}

We conducted a retrospective review of fetuses with CLHO lesions at risk for possible pulmonary venous obstruction and left atrial hypertension secondary to RAS. All data collection and storage were done under approval of the institutional review board to ensure proper handling of protected health information under the Health Information Portability and Accountability Act. The study population included infants with the prenatal diagnosis of CLHO, primarily consisting of hypoplastic left heart syndrome or doubleoutlet right ventricle with mitral atresia, studied by fetal echocardiogram between June 2004 and August 2009 who were treated at the Cincinnati Children's Hospital Medical Center. Fetuses with unbalanced atrioventricular canal defects were not included in the analysis. Serial data were collected in fetuses, 2 of which had late third trimester ratios included in our previous report, with at least 2 studies performed 4 or more weeks apart, with the initial study performed at 28 weeks or less gestation. Comparison between the initial study and the last study was performed to evaluate serial change in FR VTI ratio. Only the last study before delivery was used to assess the clinical performance of using a PVD FR VTI ratio of 5 or less.

\section{Fetal Echocardiography}

Fetal echocardiograms were performed on Sequoia C512 (Siemens Corp, Mountain View, Calif) machines under direct supervision of a cardiologist experienced in fetal echocardiography. Complete 2-dimensional, color flow, and spectral Doppler studies were performed by use of accepted standards to establish the anatomic diagnosis. ${ }^{17}$ Particular attention was devoted to evaluation of the patency of the foramen ovale by 2-dimensional and color flow Doppler.

\section{Pulmonary Venous Flow Analysis}

Pulsed-wave Doppler evaluation of pulmonary vein flow was performed in the right or left vein, because differences between the right and left pulmonary vein flow have been shown to be insignificant in the fetus. ${ }^{18}$ Pulmonary veins were imaged by color flow Doppler in the transverse plane. Doppler recordings were obtained with the angle of insonation as parallel to the direction of flow as possible with the sample volume placed in the pulmonary vein immediately proximal to its entry into the left atrium, as previously described. ${ }^{10,14,15}$ Low-pass filters were adjusted to ensure recording of lower-velocity signals. Because our previous publication ${ }^{12}$ reported interand intraobserver variability of $7 \%$ to $16 \%$ for multiple PVD measurements, including FR VTI ratios, we did not readdress that in this study.

All studies were digitally acquired and stored. Doppler measurements were made offline on commercially available digital imaging work stations. The velocity-time integral of forward flow $(\mathrm{S}+\mathrm{D}$ waves) and reverse flow (A wave) were obtained from the pulmonary venous spectral Doppler tracings, and a forward-to-reverse VTI ratio was calculated (Figure 1). All measurements were performed in triplicate and averaged to take into account beat-to-beat variation.

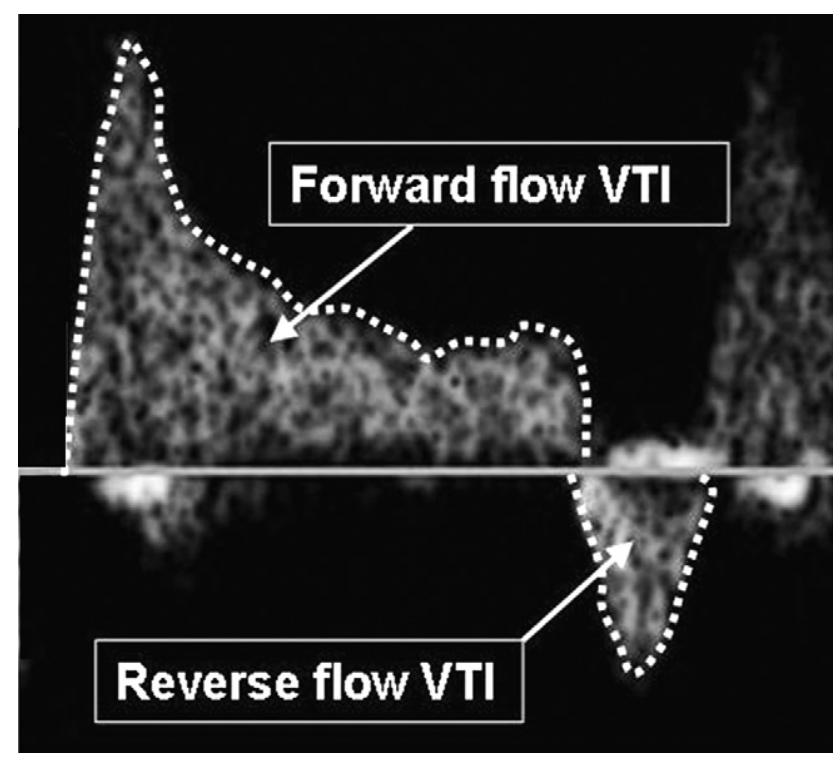

FIGURE 1. Measurement of forward-flow VTI (combined S and D waves) and reverse-flow VTI (A wave). VTI, Time-velocity integral.

\section{Diagnosis of Restrictive Atrial Septum}

An intact or highly restrictive atrial septum was suspected if the atrial septum was not freely mobile or appeared thick with limited or no demonstrable shunting on color Doppler interrogation. On the basis of our prior work, RAS was diagnosed when a small or absent interatrial communication was present and the PVD FR VTI ratio was 5 or less. ${ }^{12}$ Presence of decompressing venous channels, patent mitral valve inflow, and left ventricular size were not used as criteria in determining the likelihood of RAS.

\section{Clinical Management}

Cincinnati Children's Hospital Medical Center is a large, freestanding tertiary care pediatric center. Ambulance transfer of acutely ill neonates from all birthing centers in our referral area is therefore necessary. With these logistics in mind, 3 treatment plans were discussed in detail and offered to families when fetal RAS was identified: (1) standard delivery at an outside hospital and immediate transfer to the catheterization laboratory (cardiac intensive care unit); (2) $\mathrm{C} / \mathrm{S}$ in a hybrid catheterization laboratory/operating room for EAS; or (3) ex utero intrapartum therapy-to-extracorporeal membrane oxygenation (EXIT-to-ECMO) with EAS on ECMO. During an EXIT procedure, the head of the infant is delivered via $\mathrm{C} / \mathrm{S}$ so that ECMO support can be initiated before separation of the infant from the placenta. In all cases, the final delivery strategy decided on was individualized on the basis of consensus among the patient, maternal-fetal-medicine specialist, and cardiologist.

\section{Postnatal Data}

For the purpose of analysis, confirmation of RAS in the newborn period was used as the primary clinical outcome variable. RAS was confirmed postnatally by (1) clinical status, based on the degree of hypoxemia, acidosis, postnatal echo findings, or suspicion of significant left atrial hypertension; or (2) direct left atrial pressure measurement at cardiac catheterization (in 2 subjects undergoing EXIT-to-ECMO).

\section{Statistical Analysis}

Descriptive data are reported as mean \pm standard deviation. Nonnormal data are presented as median and range. Sensitivity, specificity, 
and positive and negative predictive values of FR VTI ratio were calculated using the predetermined cut point of 5 or less, which was based on our prior receiver operating curve analysis. ${ }^{12}$ Values for these same indices were also determined if the cut point was hypothetically lowered to 3 or less. Statistical analysis was performed using StatView v5.0 (SAS Institute Inc, Cary, NC).

\section{RESULTS}

\section{Study Population}

As in our original study, ${ }^{12}$ perinatal management was guided by PVD in 41 consecutive patients with a prenatal diagnosis of CLHO. Two infants were excluded from analysis. One infant was excluded because of the presence of hypoplastic left heart syndrome in the setting of a genetically confirmed familial cardiomyopathy with restrictive physiology. A second infant with aortic stenosis and severe mitral insufficiency was excluded because of an unusual PVD profile with a dominant $D$ wave, no visible $S$ wave, and a moderate A-wave reversal that precluded normal analysis of FR VTI ratio. The mean gestational age at last study before delivery was $33 \pm 2$ weeks. All fetuses were in a normal cardiac rhythm with 1:1 atrioventricular conduction at the time of PVD study. A total of 32 fetuses had a serial PVD assessment with a mean of $3 \pm 1$ studies. Two of these fetuses had late third trimester data included in our prior report. The serial PVD data from these 2 fetuses were included only in the analysis of longitudinal data, but not in the analysis of clinical test performance. The mean gestational age at initial fetal echocardiogram in this cohort was $24 \pm 2$ weeks.

\section{Clinical Performance of Pulmonary Venous Doppler Forward/Reverse Velocity Time Integral Ratio in Predicting Restrictive or Intact Atrial Septum}

PVD findings, clinical accuracy, and clinical outcomes for the entire study cohort are summarized in Figure 2. PVD tracings were uniformly possible in all study subjects and generally of good quality even in the setting of marginal acoustic windows.

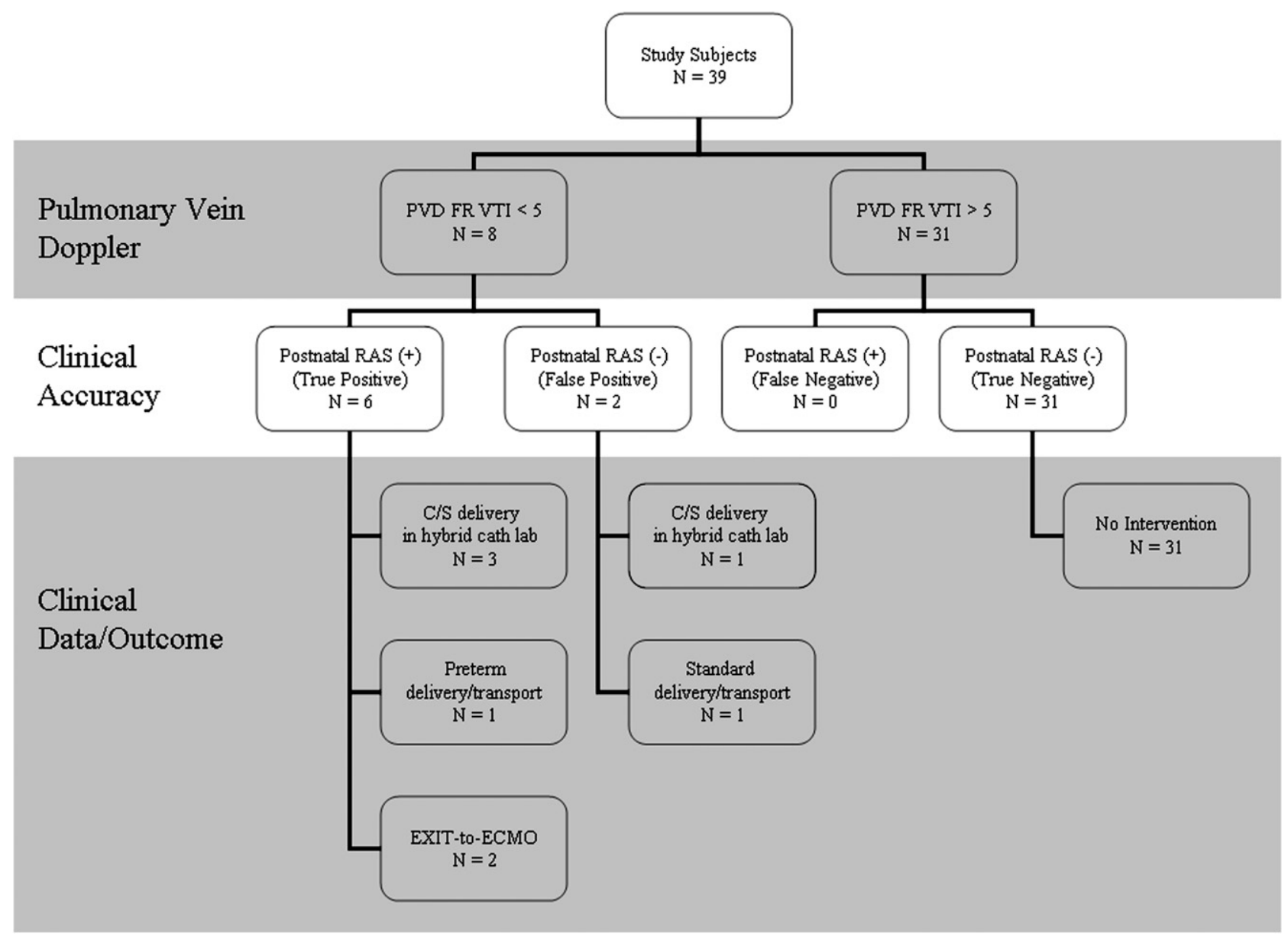

FIGURE 2. Flowchart outlining the clinical accuracy of using a PVD FR VTI ratio less than 5. PVD, Pulmonary venous Doppler; FR, forward/reverse; VTI, time-velocity integral; $R A S$, restrictive atrial septum; $C / S$, cesarean section; EXIT, ex utero intrapartum treatment; ECMO, extracorporeal membrane oxygenation. 
TABLE 1. Clinical data and immediate outcome in eight fetuses with pulmonary venous Doppler forward/reverse time-velocity integral ratios less than 5

\begin{tabular}{|c|c|c|c|c|c|c|c|}
\hline & $\begin{array}{c}\text { Last prenatal } \\
\text { FR VTI }\end{array}$ & Delivery mode & Diagnosis & $\begin{array}{c}\text { IAS on } \\
\text { postnatal study? }\end{array}$ & Intervention & Initial palliation & Mortality $<\mathbf{3 0 ~ d}$ \\
\hline \multirow[t]{6}{*}{ TP } & 1.6 & EXIT-to-ECMO & Critical AS & Yes & BAV & Aortic homograft & Yes \\
\hline & 2.2 & EXIT-to-ECMO & HLHS & No & BAS & Norwood* & No \\
\hline & 0.9 & $\mathrm{C} / \mathrm{S}$ in hybrid laboratory & HLHS & Yes & BAS/stent & Norwood* & No \\
\hline & 1 & Preterm, standard & HLHS & Yes & Failed BAS & N/A & Yes \\
\hline & 2.8 & $\mathrm{C} / \mathrm{S}$ in hybrid laboratory & HLHS & No & Surgical septectomy & Norwood* & No \\
\hline & 2.9 & $\mathrm{C} / \mathrm{S}$ in hybrid laboratory & HLHS & No & BAS & N/A & Yes \\
\hline \multirow[t]{2}{*}{ FP } & 3.6 & Standard & HLHS & No & None & Norwood w/Sano*, $\dagger$ & Yes \\
\hline & 3.5 & $\mathrm{C} / \mathrm{S}$ in fetal $\mathrm{OR}$ & HLHS & No & None & Norwood* & Yes \\
\hline
\end{tabular}

$T P$, True positive; $F P$, false positive; $F R V T I$, forward/reverse velocity time integral; $I A S$, intact atrial septum; $C / S$, cesarean section; $O R$, operating room; $E X I T$, ex utero intrapartum therapy; $E C M O$, extracorporeal membrane oxygenation; $B A V$, balloon aortic valvuloplasty; $B A S$, balloon atrial septostomy. $*$ Norwood procedure: palliative procedure used for infants with hypoplastic left heart syndrome in whom the pulmonary artery and diminutive ascending aortic are connected, homograft is used to reconstruct the aortic arch, and pulmonary blood flow is supplied by an artificial tube graft (traditionally a Blalock-Taussig shunt from the innominate artery to the right pulmonary artery). $\dagger$ Sano: shunt from the right ventricle to the right pulmonary artery (alternative to the Blalock-Taussig shunt).

Of the 39 fetuses not previously reported, 8 were identified as having RAS by the appearance of the atrial septum on 2-dimensional/color flow Doppler imaging and a PVD FR VTI ratio of 5 or less. The clinical data in these 8 fetuses are summarized in Table 1. Six fetuses required EAS/balloon valvuloplasty in the immediate newborn period. Of those 6 infants, 2 underwent EXIT-to-ECMO, 3 were delivered in the hybrid laboratory for immediate EAS, and 1 was delivered preterm in a precipitous fashion at an outside delivery hospital. The infants who underwent EXIT-to-ECMO were documented to have left atrial hypertension with mean left atrial pressures of 18 and $40 \mathrm{~mm} \mathrm{Hg}$, respectively. One infant underwent EXIT-to-ECMO and aortic balloon valvuloplasty while on ECMO support, because she was thought to have only mild left ventricular hypoplasia on postnatal echocardiography, and the clinical decision was to pursue an attempt at biventricular physiology. One infant underwent surgical septectomy in the hybrid catheterization suite immediately after unsuccessful transcatheter balloon septostomy. Ultimately, the decision to perform EAS was based on the consensus among the intensive care, echocardiography, and cardiac interventionalist personnel who were present at the time of delivery. In general, findings included severe hypoxemia, acidosis, and echocardiography confirming RAS at the time of intervention.

There were 2 false-positive results (Table 1). One infant was delivered via $\mathrm{C} / \mathrm{S}$ in the hybrid laboratory, and 1 infant was delivered in standard fashion. Postnatal echocardiography in each baby confirmed restriction of left-to-right shunting across the atrial septum with mean gradients of 10 to 12 $\mathrm{mm} \mathrm{Hg}$. Despite this, both infants had persistent oxygen saturations greater than $80 \%$ after initial neonatal resuscitation without evidence of pulmonary congestion on chest $x$-ray, difficulty with ventilation, or increased lactate levels. The Norwood procedure was performed on day 2 of life in each case.

The overall prevalence of RAS in infants with CLHO was $15 \%$ (6/39). In clinical application, a PVD FR VTI ratio of 5 or less had an overall sensitivity of $100 \%$, specificity of $94 \%$, negative predictive value of $100 \%$, and positive predictive value of $75 \%$. Lowering the cutoff value to 3 or less would have resulted in elimination of all false-positive findings in this series without an observed change in sensitivity.

\section{Serial Evaluation of Pulmonary Venous Doppler Forward/Reverse Velocity Time Integral Ratio}

Thirty-two fetuses were included in the longitudinal evaluation. The mean gestational age at the time of initial study was $24 \pm 2$ weeks. The follow-up period ranged from 4 to 17 weeks with a median of 9 weeks. Serial changes in PVD FR VTI ratio relative to cut points of 5 or less and 3 or less are presented in Figure 3. In 7 of 8 fetuses $(88 \%)$ with a PVD FR VTI ratio of 5 or less on their initial echocardiogram, the ratio remained 5 or less during late gestation and correlated with RAS postnatally in 6 of 8 . One fetus had a ratio of less than 5 over 2 studies and did not require EAS. One fetus had repeat ratios more than 5 and did not require EAS. There were 24 fetuses with ratios more than 5 on their initial echocardiogram, all of which remained more than 5 during late gestation and correlated with an unrestrictive atrial septum.

In subjects with an initial PVD FR VTI ratio of 3 or less, 5 of 6 fetuses had ratios of 3 or less on both the initial and final study (although some had intermediate values $>3$ ); all 5 went on to require postnatal EAS. One of 6 fetuses who had an initial ratio of 3 and a follow-up ratio between 3 and 5 did not require postnatal EAS. Overall, 30 of 32 $(94 \%)$ of late second trimester PVD FR VTI ratios were predictive of values obtained during the third trimester.

\section{Postnatal Findings and Outcome}

Decompressing venous channels were present postnatally in 3 of 6 subjects requiring EAS and 3 of 33 subjects with unrestrictive atrial septal defects. Three of 6 neonates who required emergency newborn intervention had an 


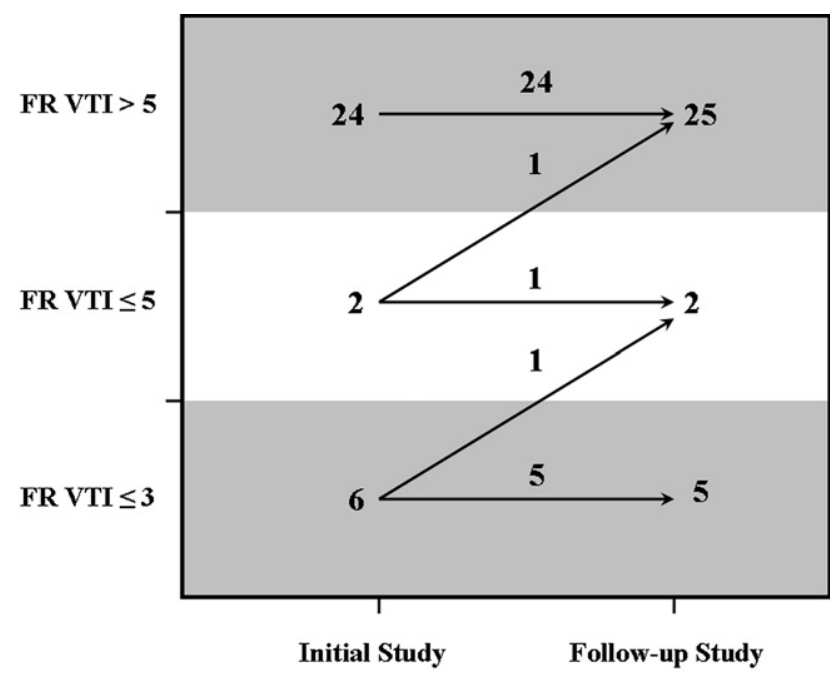

FIGURE 3. Longitudinal changes in serial FR VTI data using the initial ratio and the last ratio obtained before delivery. Numbers indicate number of subjects in each range. Note only 2 subjects had FR VTI ratios that crossed (diagonal lines) over the thresholds of 5 or less and 3 or less. $F R$, Forward/reverse; VTI, time-velocity integral.

intact atrial septum on postnatal echocardiogram, and the remaining 3 neonates had restrictive atrial level defects. Short-term follow-up was available for all infants. Thirtyday survival for infants undergoing EAS was 50\% (3/6). One infant, delivered precipitously preterm and urgently transported, died of severe hypoxemia and acidosis during attempted EAS. One infant died after EAS but before surgical intervention.

\section{DISCUSSION}

Our data demonstrate that PVD flow patterns, assessed in the late second or third trimester by fetal echocardiography, can reliably identify fetuses with CLHO who will require EAS in the newborn period. These findings establish assessment of PVD in the fetus with CLHO as an important diagnostic adjunct in planning perinatal management of these high-risk cases.

CLHO lesions compromise $15 \%$ to $20 \%$ of all congenital heart defects that are diagnosed prenatally. ${ }^{19}$ Between $6 \%$ and $20 \%$ of these infants will require EAS after birth because of an intact $(6 \%)$ or a highly restrictive $(\sim 20 \%)$ atrial septum. ${ }^{1-4}$ The overall prevalence of RAS in our study cohort is $15 \%(6 / 39)$, which is consistent with prior reports. ${ }^{1-4}$ Prenatal identification of RAS has previously been important because it allows for additional parental counseling regarding the increased morbidity and mortality observed in these infants. Accurate identification of RAS also permits more precise patient selection for either a nonstandard delivery strategy or selection for fetal cardiac intervention. ${ }^{6,9}$

However, despite prenatal identification of RAS and the implementation of a perinatal strategy to allow for prompt

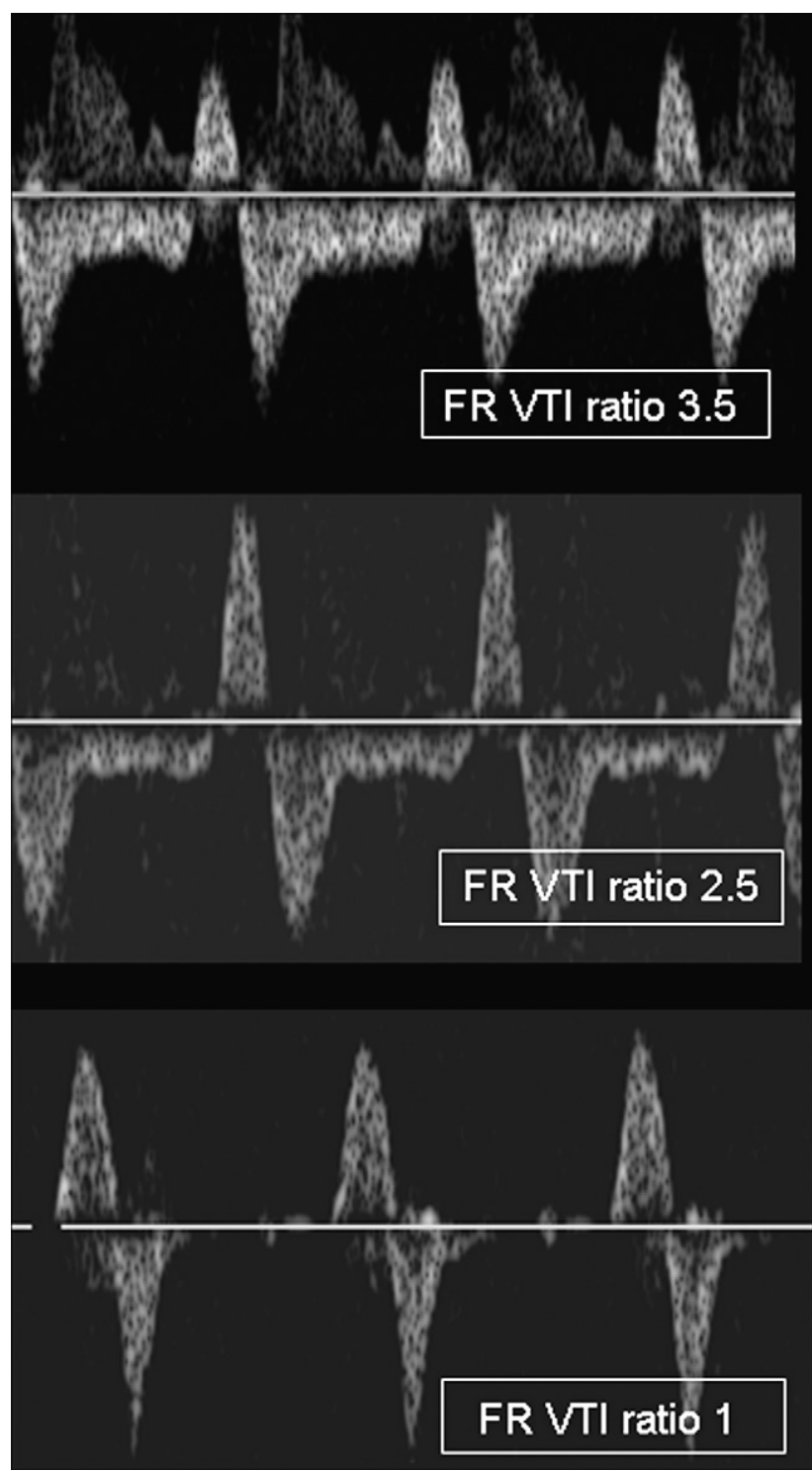

FIGURE 4. Pulmonary venous Doppler profiles in 3 fetuses with CLHO who required EAS in the newborn period. Note the variability in the FR VTI ratios. FR, Forward/reverse; VTI, time-velocity integral.

cardiology evaluation and treatment, infants with CLHO lesions complicated by RAS still have poor short- and long-term outcomes. ${ }^{1,2,20,21}$ This is supported by our current series in which 30-day survival was only $50 \%$, despite the use of novel strategies such as EXIT-to-ECMO and cesarean delivery in the hybrid catheterization suite. Poor short-term survival despite prenatal diagnosis of RAS has prompted select centers to perform in utero atrial septoplasty to relieve left atrial hypertension, thus, theoretically preventing the irreversible changes to the pulmonary vasculature.

The feasibility of a novel delivery strategy or in utero intervention depends not only on the ability to execute these interventions but also on the ability to accurately predict 


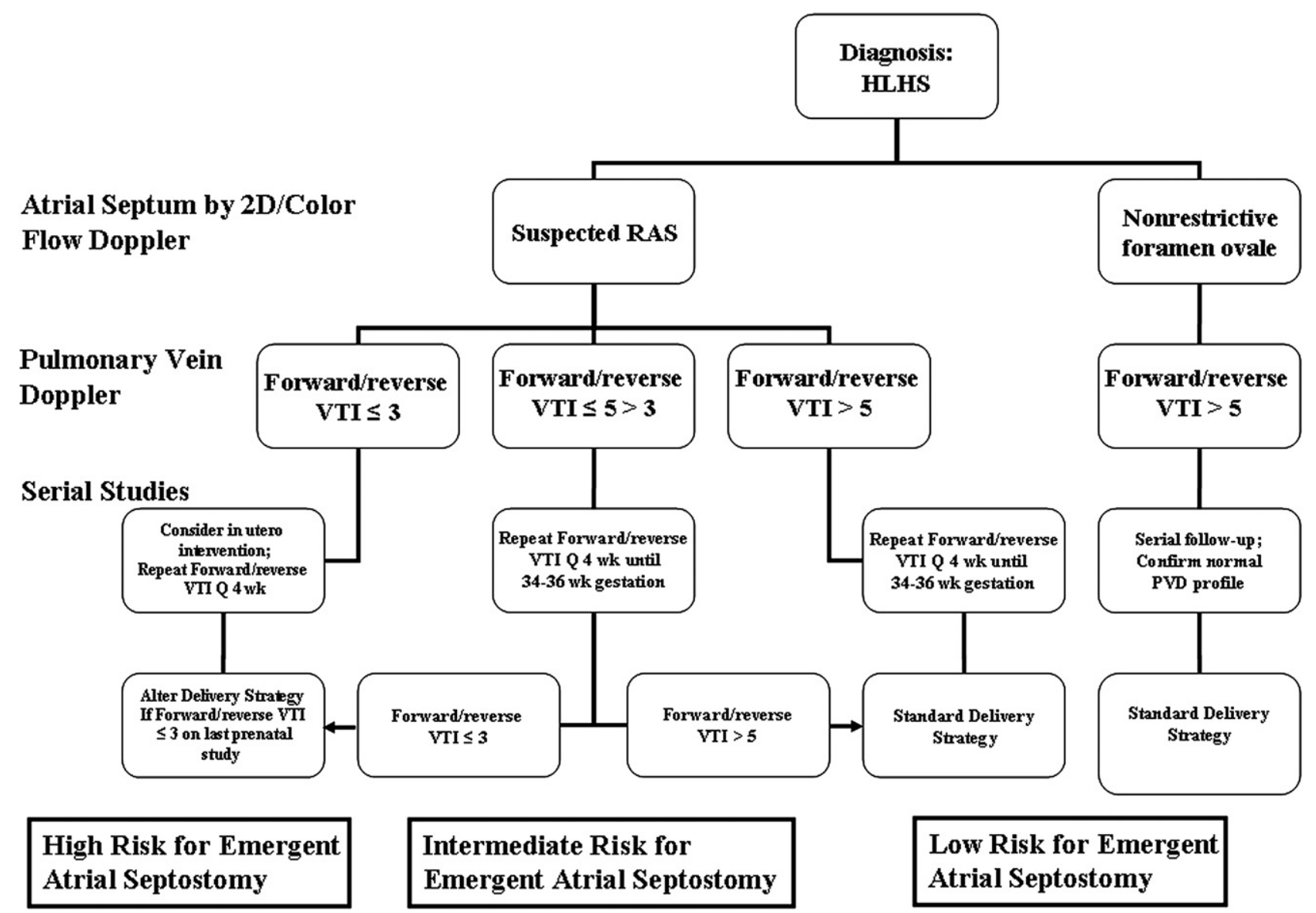

FIGURE 5. Proposed algorithm to assist clinicians in diagnosing RAS and determining the probability of postnatal EAS. RAS, Restrictive atrial septum; $V T I$, time-velocity integral; EAS, emergency atrial septostomy.

early in gestation which infants will require EAS. The current study demonstrates that PVD flow patterns, in the setting of a small or absent interatrial communication, are able to reliably identify fetuses with CLHO who will require EAS, both in late second and third trimesters. Although our previous analysis of PVD patterns had suggested that using an FR VTI ratio less than 5 was the strongest predictor of RAS in the newborn period, our subsequent experience suggests that lowering the PVD FR VTI ratio threshold to 3 or less would have increased specificity and PPV. Although changing the threshold value in this way may affect sensitivity, this may, nonetheless, be a preferable strategy when considering maternal interventions that add risk of morbidity to the mother.

Within the current study cohort, we again noted the variability of fetal pulmonary venous spectral Doppler patterns that correlate with RAS on postnatal evaluation (Figure 4). Specifically, not every neonate with CLHO and confirmed RAS will have the biphasic fetal PVD profile-consisting of a large $\mathrm{S}$ wave, absent $\mathrm{D}$ wave, and large A-wave reversal-described as the "type C" pattern by Taketazu and colleagues. ${ }^{13}$ This pattern would generally produce a PVD FR VTI ratio of approximately 1 and correlated in their study to fetuses with intact atrial septum. Our findings demonstrate that EAS is not uncommonly required in patients with small interatrial communications and PVD FR VTI ratios of 3 or less, which broadens the scope of "at risk" fetuses identified using our proposed threshold values.

Review of the longitudinal data demonstrated that 30 of $32(94 \%)$ late second-trimester PVD FR VTI ratios did not change significantly with respect to the threshold levels when compared with those obtained during the later third trimester. In 5 of 6 fetuses with an FR VTI ratio 3 or less at initial study, the ratio remained 3 or less on follow-up study and correlated postnatally with confirmed RAS. These findings have implications for patient selection for fetal atrial septoplasty, because these procedures are usually undertaken in the late second trimester in an effort to affect the development of pulmonary vascular changes. ${ }^{9,22}$ These findings have led us to consider an algorithmic approach to evaluation of the fetus with CHLO at risk of RAS (Figure 5). On the basis of our most recent experience, our approach has evolved toward recommending $\mathrm{C} / \mathrm{S}$ in the cardiac catheterization laboratory for FR VTI less than 
3. This strategy typically results in a low-transverse incision that does not preclude attempts at a vaginal birth in subsequent pregnancies. EXIT-to-ECMO, on the other hand, requires deep maternal anesthesia to control uterine tone and often requires a vertical incision, which increases the risk to the mother during the current and possibly future deliveries.

Limitations to this study include the small sample size and retrospective study design, which may limit our ability to generalize our findings. We also acknowledge that management of high-risk infants such as these is likely institution or provider dependent, which may make it more or less likely that an infant with the same clinical presentation would undergo EAS.

\section{CONCLUSIONS}

Because the mother incurs added risks when in utero or alternate delivery strategies are implemented, we believe that using a PVD FR VTI ratio of 3 or less allows for identification of infants with a high likelihood of requiring EAS in the newborn period while eliminating false-positive results. In those fetuses with ratios between 3 and 5 in whom it is more difficult to predict postnatal outcome, we would recommend serial evaluations and a more cautious prenatal counseling approach with regard to the strategy for delivery and our ability to accurately predict the need for EAS in the newborn period. Overall, the results of the longitudinal data set suggest that the PVD FR VTI ratios obtained in late second trimester are consistent with values observed at later gestation and, as such, could be reliably used for patient selection for in utero interventions, particularly in fetuses with an FR VTI ratio of 3 or less.

The authors thank Yu Wang, MS, for expertise and input regarding the statistical analysis used in this article.

\section{References}

1. Rychik J, Rome JJ, Collins MH, DeCampli WM, Spray TL. The hypoplastic left heart syndrome with intact atrial septum: atrial morphology, pulmonary vascular histopathology and outcome. J Am Coll Cardiol. 1999;34:554-60.

2. Vlahos AP, Lock JE, McElhinney DB, van der Velde ME. Hypoplastic left heart syndrome with intact or highly restrictive atrial septum: outcome after neonatal transcatheter atrial septostomy. Circulation. 2004;109:2326-30.

3. Atz AM, Feinstein JA, Jonas RA, Perry SB, Wessel DL. Preoperative management of pulmonary venous hypertension in hypoplastic left heart syndrome with restrictive atrial septal defect. Am J Cardiol. 1999;83:1224-8.
4. Vida VL, Bacha EA, Larrazabal A, Gauvreau K, Thiagaragan R, FynnThompson F, et al. Hypoplastic left heart syndrome with intact or highly restrictive atrial septum: surgical experience from a single center. Ann Thorac Surg. 2007;84:581-5.

5. Glatz JA, Tabbutt S, Gaynor JW, Rome JJ, Montenegro L, Spray TL, et al. Hypoplastic left heart syndrome with atrial level restriction in the era of prenatal diagnosis. Ann Thorac Surg. 2007;84:1633-8.

6. Michelfelder E, Polzin W, Hirsch R. Hypoplastic left heart syndrome with intact atrial septum: utilization of a hybrid catheterization facility for cesarean section delivery and prompt neonatal intervention. Catheter Cardiovasc Interv. 2008;72: 983-7.

7. Cheatham JP. Intervention in the critically ill neonate and infant with hypoplastic left heart syndrome and intact atrial septum. J Intervent Cardiol. 2001;14: 357-66.

8. Vance MS. Hypoplastic left heart syndrome with intact atrial septum: levoatriocardinal vein stent placement as a bridge to surgery. Catheter Cardiovasc Interv. 2002;57:85-7.

9. Marshall AC, Levine J, Morash D, Silva V, Lock JE, Benson CB, et al. Results of in utero atrial septoplasty in fetuses with hypoplastic left heart syndrome. Prenat Diagn. 2008;28:1023-8.

10. Better DJ, Apfel HD, Zidere V, Allan LD. Pattern of pulmonary venous blood flow in the hypoplastic left heart syndrome in the fetus. Heart. 1999;81:646-9.

11. Lenz F, Chaoui R. Changes in pulmonary venous Doppler parameters in fetal cardiac defects. Ultrasound Obstet Gynecol. 2006;28:63-70.

12. Michelfelder E, Gomez C, Border W, Gottliebson W, Franklin C. Predictive value of fetal pulmonary venous flow patterns in identifying the need for atrial septoplasty in the newborn with hypoplastic left ventricle. Circulation. 2005;112:2974-9.

13. Taketazu M, Barrea C, Smallhorn JF, Wilson GJ, Hornberger LK. Intrauterine pulmonary venous flow and restrictive foramen ovale in fetal hypoplastic left heart syndrome. J Am Coll Cardiol. 2004;43:1902-7.

14. Better DJ, Kaufman S, Allan LD. The normal pattern of pulmonary venous flow on pulsed Doppler examination of the human fetus. J Am Soc Echocardiogr. 1996;9:281-5

15. Lenz F, Chaoui R. Reference ranges for Doppler-assessed pulmonary venous blood flow velocities and pulsatility indices in normal human fetuses. Prenat Diagn. 2002;22:786-91.

16. Paladini D, Palmieri S, Celentano E, Guida F, Salviati M, Morra T, et al. Pulmonary venous blood flow in the human fetus. Ultrasound Obstet Gynecol. 1997;10: $27-31$.

17. Rychik J, Ayres N, Cuneo B, Gotteiner N, Hornberger L, Spevak PJ, et al. American Society of Echocardiography guidelines and standards for performance of the fetal echocardiogram. J Am Soc Echocardiogr. 2004;17:803-10.

18. Crowe DA, Allan LD. Patterns of pulmonary venous flow in the fetus with disease of the left heart. Cardiol Young. 2001;11:369-74.

19. Allan LD, Sharland GK, Milburn A, Lockhart SM, Groves AM, Anderson RH, et al. Prospective diagnosis of 1,006 consecutive cases of congenital heart disease in the fetus. J Am Coll Cardiol. 1994;23:1452-8.

20. Gaynor JW, Mahle WT, Cohen MI, et al. Risk factors for mortality after the Norwood procedure. Eur J Cardiothorac Surg. 2002;22:82-9.

21. Atz AM, Travison TG, Williams IA, Pearson GD, Laussen PC, Mahle WT, et al. Prenatal diagnosis and risk factors for preoperative death in neonates with single right ventricle and systemic outflow obstruction: screening data from the Pediatric Heart Network Single Ventricle Reconstruction Trial. J Thorac Cardiovasc Surg. 2010;140:1245-50.

22. Marshall AC, van der Velde ME, Tworetzky W, Gomez CA, Wilkins-Haug L, Benson CB, et al. Creation of an atrial septal defect in utero for fetuses with hypoplastic left heart syndrome and intact or highly restrictive atrial septum. Circulation. 2004;110:253-8. 